\title{
Síntese, caracterização de complexos de Base de Schiff com níquel (II) ancorados no material mesoporoso SBA-15.
}

\author{
Danielle de Oliveira Maia (Profa. na Universidade Federal do Maranhão - UFMA) \\ Aruzza Mabel de Morais Araújo (Doutoranda pela Engenharia do Petróleo da Universidade Federal \\ do Rio Grande do Norte - UFRN) \\ Ana Paula de Melo Alves Guedes (Profa. do Instituto de Quimica na Universidade do Estado do \\ Rio Grande do Norte - UFRN)
}

Amanda Duarte Gondim (Profa. do Instituto de Química na Universidade do Estado do Rio Grande do Norte -UFRN)

Antonio Souza de Araujo (Prof. do Instituto de Química na Universidade do Estado do Rio Grande do Norte -UFRN)

Francisco das Chagas Dantas de Lemos (Prof. do Instituto de Química na Universidade Federal de Tocantis -Araguaína- UFTO)

Edward Ralph Dockal (Prof. do Departamento de Química na Universidade Federal de São

Carlos - UFSCAR)

*E-mail: dany_ufrn@yahoo.com.br

Resumo

SBA-15 são materiais mesoporosos, que possuem uma rede de canais e poros de tamanho bem definido na escala nanométrica, além de outras características, tais como, elevada estabilidade térmica e área superficial. Essa arquitetura particular de poros torna esses materiais promissores na área de ancoramento de uma variedade de compostos na matriz da sílica resultando em aplicações nas diversas áreas, dentre elas, na catálise. Nesse trabalho, foram sintetizados complexos de Base de Schiff com níquel (II) para serem ancorados na SBA-15 funcionalizada com 3-cloropropiltrimetóxisilano realizando um estudo da estabilidade térmica desses compostos. Após a síntese dos complexos, estes foram caracterizados por análise elementar (CHN), espectroscopia de absorção na região do infravermelho e análises térmicas (TG/DTG). A análise elementar sugere que os complexos apresentam as seguintes fórmulas químicas gerais: [ $\left.\mathrm{Ni}\left(\mathrm{C}_{18} \mathrm{H}_{19} \mathrm{~N}_{3} \mathrm{O}_{2}\right)\right] \cdot 2 \mathrm{CH}_{3} \mathrm{COO} . \mathrm{H}_{2} \mathrm{O}$, [ $\left.\mathrm{Ni}\left(\mathrm{C}_{20} \mathrm{H}_{23} \mathrm{~N}_{3} \mathrm{O}_{2}\right)(2 \mathrm{Cl})\right] .2 \mathrm{H}_{2} \mathrm{O}$, sendo $\mathrm{L} 1=$ $\mathrm{C}_{18} \mathrm{H}_{19} \mathrm{~N}_{3} \mathrm{O}_{2}, \mathrm{~L} 2=\mathrm{C}_{20} \mathrm{H}_{23} \mathrm{~N}_{3} \mathrm{O}_{2}$. Após caracterização dos complexos, confirmando a coordenação metal - ligante, estes foram ancorados no material mesoporoso. A caracterização desses materiais foi realizada por difração de raios - X, adsorção e dessorção de $\mathrm{N}_{2}$ e análises térmicas (TG/DTG). No DRX foram observados três picos principais de difração, cujos índices de Miller são (100), (110) e (200), mostrando que mesmo após a ancoragem, os materiais mesoporosos não perderam suas características estruturais. Através da adsorção e dessorção de $\mathrm{N}_{2}$, foram observadas que os materiais apresentaram isoterma do tipo IV e histerese do tipo $\mathrm{H} 1$ característicos dos materiais mesoporos. Na espectroscopia de absorção na região do infravermelho, os materiais ancorados apresentaram bandas características dos ligantes (Base de Schiff) e da sílica evidenciando o sucesso da ancoragem. Na análise térmica, foram observadas a decomposição de água adsorvida, água coordenada, aminas, aromáticos, ligantes, cloropropiltrimetóxisilano e também um aumento na estabilidade térmica (eliminação dos ligantes) das sílicas ancoradas [Ni(L1)]SBA-15 e [Ni(L2)]SBA-15 quando comparado aos complexos livres, evidenciando o sucesso da ancoragem dos complexos na peneira molecular. 
Palavras - chave: SBA-15; Base de Schiff; Materiais Mesoporosos; Termogravimetria; DRX.

Abstract

SBA-15 materials are mesoporous, having a network of channels and well defined pore size in the nanometer range, as well as other features such as high thermal stability and surface area. This particular pore architecture makes these promising materials in the anchoring area of a variety of compounds in the silica matrix resulting in applications in several fields, among them, in catalysis. In this work, they synthesized Schiff Base complexes of nickel (II) to be anchored in the SBA-15 functionalized with 3-chloropropyltrimethoxysilane conducting a study of thermal stability of these compounds. After synthesis of the complexes, they were characterized by elemental analysis (CHN), absorption spectroscopy in the infrared region and thermal analysis (TG / DTG). Elemental analysis suggests that the complexes have the following general chemical formulas: $\quad\left[\mathrm{Ni}\left(\mathrm{C}_{18} \mathrm{H}_{19} \mathrm{~N}_{3} \mathrm{O}_{2}\right)\right] 2 \mathrm{CH}_{3}$ COO. $\mathrm{H}_{2} \mathrm{O}, \quad\left[\mathrm{Ni}\left(\mathrm{C}_{20} \mathrm{H}_{23} \mathrm{~N}_{3} \mathrm{O}_{2}\right)(2 \mathrm{Cl})\right] 2 \mathrm{H}_{2} \mathrm{O}$, being $\mathrm{C}_{18} \mathrm{H}_{19} \mathrm{~N}_{3} \mathrm{O}_{2}=\mathrm{L} 1, \mathrm{~L} 2=\mathrm{C}_{20} \mathrm{H}_{23} \mathrm{~N}_{3} \mathrm{O}_{2}$. After characterization of the complex, confirming the coordinating metal - ligand, these were anchored in the mesoporous material. The characterization of these materials was carried out by diffraction of $\mathrm{X}$ - ray adsorption and desorption of $\mathrm{N}_{2}$ and thermal analysis (TG / DTG). XRD analysis revealed three main diffraction peaks, whose Miller indices are (100), (110) and (200), showing that even after the anchoring, the mesoporous materials do not lose their structural characteristics. By $\mathrm{N}_{2}$ adsorption and desorption it was observed that the materials had type IV isotherm and type H1 hysteresis characteristic of mesoporous materials. In absorption spectroscopy in the infrared region, the materials showed characteristic bands of docked ligand (Schiff Base) and silica demonstrating the success of the anchor. In the thermal analysis, there were observed the decomposition of adsorbed water, coordinated water, amines, aromatics, ligands, chloropropyltrimethoxysilane, and also an increase in thermal stability (removal of ligands) of the silicas anchored [Ni(L1)] SBA-15 and [Ni(L2)] SBA-15 compared to free complexes, demonstrating the successful anchoring of complex molecular sieve.

Key - words: SBA-15; Schiff base; Mesoporous materials; thermogravimetry; DRX.

\section{Introdução}

A SBA-15 (Santa Barbara Amorphous) sintetizada na década de 90 na Universidade de Santa Bárbara na Califórnia é uma peneira molecular de sílica sintetizada usando o ortosilicato de tetraetila (TEOS), como fonte de sílica e o copolímero tribloco (PEO-PPO-PEO) poli-(oxido propileno)-poli-(oxido etileno) como agente direcionador de estrutura (molde). Possui microporos formados pela penetração do óxido de etileno, proveniente do surfactante P123, nas paredes da sílica. Este material contém mesoporos de arranjos hexagonais uniformes e paredes espessas, o que proporciona uma maior estabilidade térmica e hidrotérmica ao material [WHANG et al 2015., VEISI et al 2014., ZHAO et al., 1998a., ZHAO et al., 1998b]. Os poros bem definidos fazem da SBA-15 um material muito promissor na área de ancoragem de uma variedade de compostos [SILVA et al., 2011], dentre eles complexos de Base de Schiff.

Bases de Schiff resultam da condensação de aminas primárias com compostos que possuam grupo carbonila ativo (aldeído ou cetonas), sendo reportada pelo químico alemão Hugo Schiff em 1864. São ligantes estruturalmente bem diversificados por possuírem nitrogênio e oxigênio como átomos doadores, podendo em alguns casos serem encontrados átomos de enxofre, como fontes de elétrons livres.

A química da Base de Schiff vem sendo uma área de interesse crescente devido à sua flexibilidade sintética, pelo fato de estabilizarem os diferentes metais em diversos estados de 
oxidação, por apresentarem propriedades estéricas e eletrônicas e devido à variedade de aplicações que esse material apresenta, em várias áreas, na biologia, na medicina e na catálise em reações de epoxidação de cis olefinas, reações de polimerização, decomposição de $\mathrm{H}_{2} \mathrm{O}_{2}$, dentre outras (adsorção, oxidação de sulfetos...) [GHUA et al., 2013., ABO-ALY et al., 2015., GRIVANI et al., 2012]. Esse complexo que possui metais de transição em sua estrutura é bem conhecido pelas suas atividades catalíticas na oxidação, bem como, redução de substratos orgânicos e para a síntese de produtos químicos finos.

Portanto a ancoragem desses complexos em suportes sólidos fornece características, tais como, seletividade, um aumento na estabilidade térmica do material e capacidade de regeneração [SHERINGTON et al., 1998].

Este trabalho tem como intuito sintetizar materiais híbridos (inorgânico-orgânico) utilizando a SBA-15 como suporte e complexos de Base de Schiff com níquel (II). Além de realizar um estudo da estabilidade térmica através das análises térmicas (TG/DTG) dos materiais obtidos antes e após de serem ancorados na sílica. A combinação das propriedades do componente orgânico com o conteúdo inorgânico forma um material atraente do ponto de vista científico pela possibilidade de combinar as funcionalidades da química orgânica com as vantagens de um composto inorgânico termicamente estável

\section{Experimental}

\subsection{Síntese da SBA-15}

A SBA-15 é sintetizada em meio forte ácido, utilizando o tetraetilortosilicato (TEOS) como fonte de sílica e o copolímero tribloco P123 $\left(\mathrm{EO}_{20} \mathrm{PO}_{70} \mathrm{EO}_{20}\right)$ como direcionador [Zhao et al,. 1998].

\subsection{Funcionalização da SBA-15 com cloropropiltrimetóxisilano}

Inicialmente $3 \mathrm{~g}$ da sílica mesoporosa (SBA-15) foram refluxadas com 2,0 $\mathrm{ml}$ de 3cloropropiltrimetóxisilano em 40,0 ml de xileno por $24 \mathrm{~h}$. Em seguida o produto foi lavado sucessivamente com xileno, etanol, acetona e éter etílico, para remover o excesso do material orgânico que não reagiu, e mantido em uma estufa à vácuo por 18 h.

\subsection{Síntese dos complexos}

Para a formação da Base de Schiff: 10 mmol de salicilaldeído foram solubilizados em 60 $\mathrm{mL}$ de clorofórmio sob agitação e aquecimento a $50^{\circ} \mathrm{C}$. Em seguida, $5 \mathrm{mmol}$ da triamina (dietilenotriamina) foi adicionada lentamente deixando a mistura reagir por 1 hora. Após esta etapa, para a formação do [Ni(L1)], adicionou-se $5,2 \mathrm{~g}$ de $\mathrm{NiCl}_{2} \cdot 6 \mathrm{H}_{2} \mathrm{O}$ e $5,2 \mathrm{~g}$ de acetato de sódio. Para o complexo [Ni(L2)], a triamina utilizada na síntese foi a 3,3' iminobispropilamina. Após a etapa da formação da base de Schiff foram adicionados $(1,18 \mathrm{~g})$ de cloreto de níquel. Os complexos foram filtrados e lavados com etanol e água destilada. Por fim os sólidos resultantes foram secos em um dessecador sob sílica gel por 5 dias.

\subsection{Ancoragem dos complexos na SBA-15}

Na síntese abaixo, foram ancorados no material mesoporoso os complexos [Ni(L1)], [Ni(L2)] e [Ni(L3)] utilizando a mesma rota sintética para cada complexo. Inicialmente 1 grama de SBA-15-Cl e 0,2 gramas dos complexos, foram deixados em suspensão em $50 \mathrm{~mL}$ de tolueno em refluxo por 24 horas. Após esta etapa, o catalisador foi separado em um extrator Soxhlet (24 horas) com tolueno para remover o excesso do complexo na superfície externa da SBA-15. Em seguida, os compostos foram lavados com água e etanol para remover o complexo que não foi ancorado e levado para uma estufa (50

$\left.{ }^{\circ} \mathrm{C}\right)$ por 24 horas. Por fim, o catalisador foi levado para uma estufa a vácuo por 5 horas (50

${ }^{\circ} \mathrm{C}$ ), figura 19 . O átomo de cloro $(\mathrm{Cl})$ presente na estrutura do material proveniente da 
funcionalização com 3-cloropropiltrimetóxisilano, ligado covalentemente, por ser muito reativo, foi facilmente substítuido por bases mais fortes através de um ataque nucleofílico por parte do nitrogênio da Base de Schiff [ALAVI et al., 2013].

\section{Resultados e Discussão}

\subsection{Análise Elementar (CHN)}

De acordo com os cálculos da análise elementar (CHN), Tabela 1, foi observado que os valores experimentais obtidos para os complexos são coerentes com os valores teóricos. Assim, a partir dos resultados obtidos pode - se confirmar as fórmulas moleculares e as estruturas propostas para os complexos. Para o primeiro complexo: [ $\left.\mathrm{Ni}\left(\mathrm{C}_{18} \mathrm{H}_{19} \mathrm{~N}_{3} \mathrm{O}_{2}\right)\right] .2 \mathrm{CH}_{3} \mathrm{COO} . \mathrm{H}_{2} \mathrm{O}$, sendo $\mathrm{L} 1=\mathrm{C}_{18} \mathrm{H}_{19} \mathrm{~N}_{3} \mathrm{O}_{2}$ e para o segundo complexo, [ $\left.\mathrm{Ni}\left(\mathrm{C}_{20} \mathrm{H}_{23} \mathrm{~N}_{3} \mathrm{O}_{2}\right)(2 \mathrm{Cl})\right]$. $2 \mathrm{H}_{2} \mathrm{O}$, em que L2= $\mathrm{C}_{20} \mathrm{H}_{23} \mathrm{~N}_{3} \mathrm{O}_{2}$.

Tabela 1. Dados de porcentagens de carbono, hidrogênio e nitrogênio nos complexos.

\begin{tabular}{lllc}
\hline Complexos & $\% \mathrm{C}$ & $\% \mathrm{H}$ & $\% \mathrm{~N}$ \\
& Teor/exp & Teor/exp & Teor/exp \\
\hline$[\mathrm{Ni}(\mathrm{L} 1)] \cdot 2 \mathrm{CH}_{3} \mathrm{COO} \cdot \mathrm{H}_{2} \mathrm{O}$ & $52,41 / 52,52$ & $5,3 / 5,14$ & $8,3 / 8,6$ \\
{$[\mathrm{Ni}(\mathrm{L} 2)(2 \mathrm{Cl})] .2 \mathrm{H}_{2} \mathrm{O}$} & $47,93 / 47,69$ & $5,19 / 5,55$ & $8,4 / 8,9$ \\
\hline
\end{tabular}

\subsection{Difração de Raios X}

Os difratogramas de raios - $\mathrm{X}$ dos materiais, Figura 1, obtidos foram utilizados para identificação da estrutura hexagonal característica dos materiais mesoporosos tipo SBA-15. Foram observados três picos principais de difração, referentes aos planos cristalográficos, cujos índices de Miller são (100), (110) e (200). As resoluções dos picos sofreram redução com pequenos deslocamentos para ângulos $2 \theta$ em comparação com a SBA-15, o que pode estar relacionado com o menor grau de espalhamento da radiação devido a maior interação entre as paredes da sílica e o composto ancorado [GALLO., 2012].
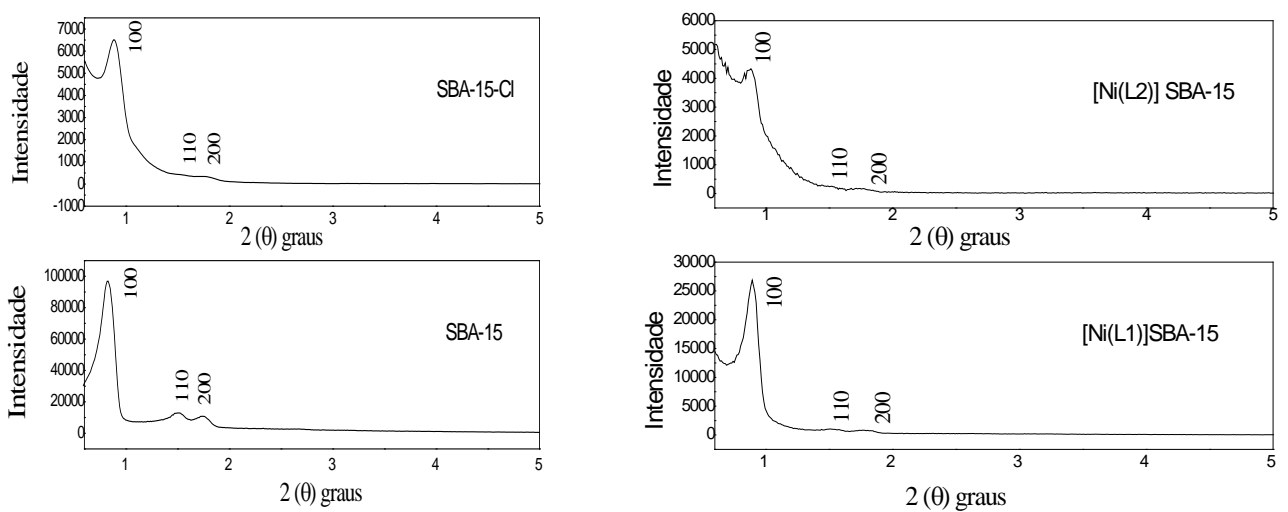

Figura 1- DRX dos materiais sintetizados.

\subsection{Adsorção e dessorção de $\mathbf{N}_{2}$}

A tabela 2 apresenta as modificações nos materiais. A área superficial, diâmetro e volume de poro dos materiais diminuíram com o decorrer das mudanças na SBA-15. Isto sugere a presença do material volumoso no interior dos poros da sílica evidenciando uma indicação clara 
de eficiência no processo tanto de funcionalização com 3-CPTMS como ancoragem com os complexos no interior do sistema de poros do material [Alavi et al., 2013].

Tabela 2 - Propriedades texturais dos materiais sintetizados.

\begin{tabular}{l|c|c|c}
\hline Materiais & $\begin{array}{c}\text { Área } \\
\text { específica } \\
\left(\mathrm{m}^{2} / \mathrm{g}\right)\end{array}$ & $\begin{array}{c}\text { Volume total } \\
\text { dos poros } \\
\left(\mathrm{cm}^{3} / \mathrm{g}\right)\end{array}$ & $\begin{array}{c}\text { Diâmetro de } \\
\text { poros } \\
(\mathrm{nm})\end{array}$ \\
\hline SBA-15 & 517,47 & 0,74 & 6,18 \\
SBA-15-Cl & 333,4 & 0,46 & 5,08 \\
Ni(L1)]SBA-15 & 325,91 & 0,50 & 5,62 \\
[Ni(L2)]SBA-15 & 296,44 & 0,45 & 5,61 \\
\hline
\end{tabular}

\subsection{Espectro de absorção na região do infravermelho}

Nos espectros da SBA-15, SBA-15 funcionalizada com 3-cloropropiltrimetóxisilano, [Ni(L1)] SBA-15, [Ni(L2)]SBA-15 e [Ni(L3)]SBA-15 foram observadas bandas abaixo de 3470 $\mathrm{cm}^{-1}$ referentes às vibrações de estiramentos dos grupos hidroxilas internos e externos na estrutura mesoporosa. Em 1113 a $1085 \mathrm{~cm}^{-1}$ estão os estiramentos assimétricos das ligações Si-O-Si. Em 808, 800, 810, 795 e $798 \mathrm{~cm}^{-1}$ os estiramentos simétricos Si-O-Si e 468, 465, 465, 454 e $462 \mathrm{~cm}^{-}$ ${ }^{1}$ as deformações Si-O-Si, respectivamente. No espectro da SBA funcionalizada, a reação entre Si-OH e o cloropropiltrimetóxisilano foi evidenciada com o decréscimo na intensidade da banda de absorção em $3469 \mathrm{~cm}^{-1}$ e a presença da banda em $2954 \mathrm{~cm}^{-1}$ referente ao estiramento C-H comprovando a funcionalização da sílica [ALAVI et al., 2013].

É importante ressaltar que para os materiais [Ni(L1)]SBA-15 e [Ni(L2)]SBA-15, foram observadas bandas características dos complexos quando comparadas com a matriz de sílica pura. A presença da estrutura orgânica proveniente dos complexos foi confirmada através da banda na região de 2991 a $2958 \mathrm{~cm}^{-1}$, atribuídas a deformações axiais $\mathrm{CH}$. Além das bandas referentes à deformação axial C=C entre 1472 a $1438 \mathrm{~cm}^{-1}$ e C=N em 1643 a $1630 \mathrm{~cm}^{-1}$.
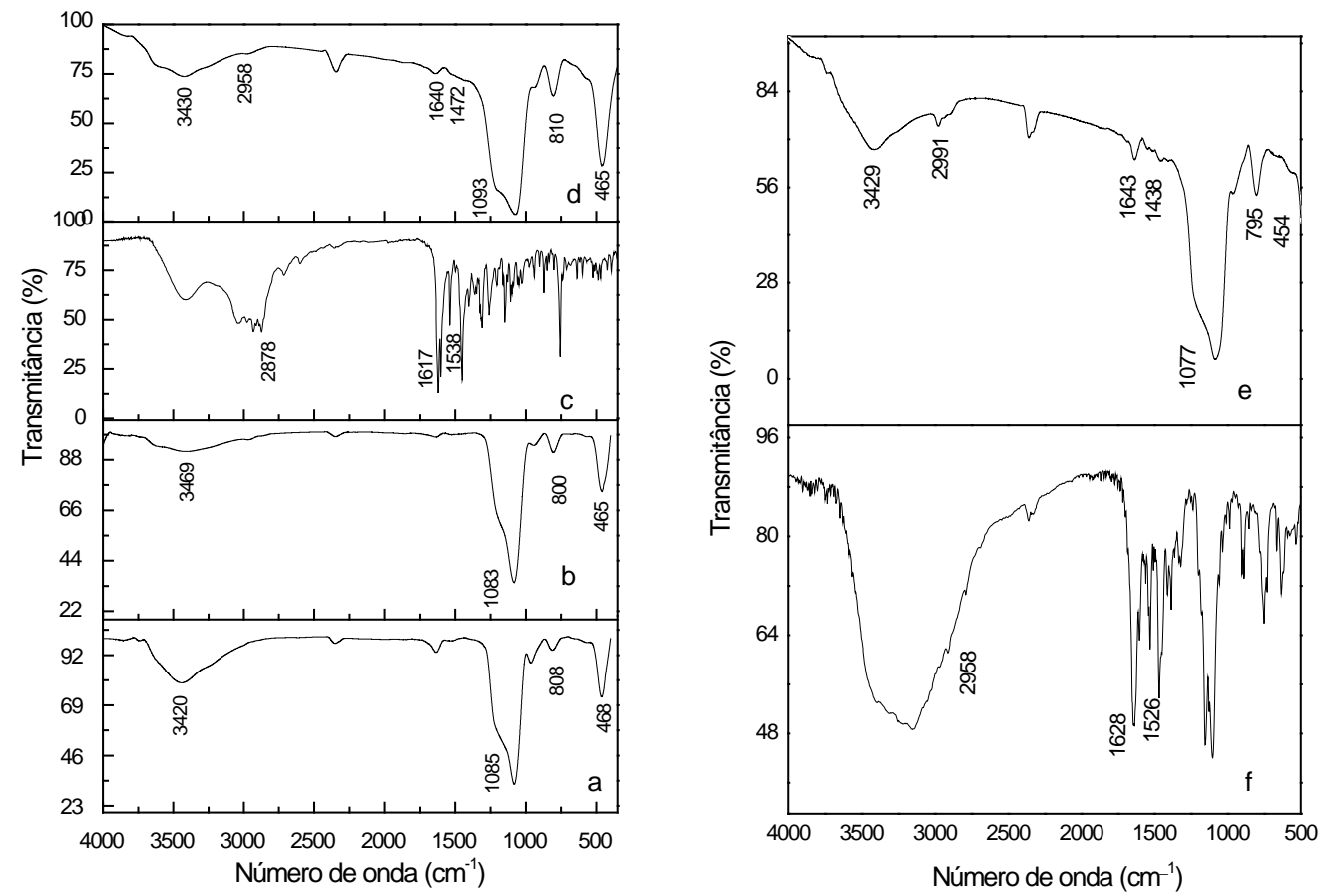

Figura 4 - Espectros de infravermelho: (a) SBA-15, (b) SBA-15-Cl, (c) [Ni(L1)], (d) [Ni(L1)SBA15, (e) $[\mathrm{Ni}(\mathrm{L} 2)](\mathrm{f})[\mathrm{Ni}(\mathrm{L} 2)] \mathrm{SBA}-15$. 


\subsection{Análise Termogravimétrica (TG/ DTG)}

A figura 2 apresenta as curvas termogravimétricas TG/DTG dos complexos [Ni(L1)], [Ni(L2)], SBA-15, SBA-15-Cl e [Ni(L1)]SBA-15 e [Ni(L2)]SBA-15. A primeira etapa de decomposição do complexo [Ni(L1)] ocorreu abaixo de $60{ }^{\circ} \mathrm{C}$ com perda de massa 2,3\% sendo atribuída a eliminação da água de hidratação [DAVID et al., 1992., YANG et al., 2011]. A segunda etapa de decomposição do complexo foi observada em $338{ }^{\circ} \mathrm{C}$ com perda de massa $(8,4 \%)$ referente ao início da decomposição da amina, acetato (confirmado pela análise elementar) e aromáticos, a terceira etapa é atribuída à decomposição térmica da amina em $362{ }^{\circ} \mathrm{C}$ e a quarta etapa em $489^{\circ} \mathrm{C}$ atribuída à eliminação do ligante. No complexo [Ni(L2)] foram observados cinco eventos: o primeiro evento em $52^{\circ} \mathrm{C}$, com a eliminação da água de hidratação [DAVID et al., 1992]. Em $134{ }^{\circ} \mathrm{C}$, o segundo evento, perda da molécula de água coordenada. Na literatura para complexos de cobalto com a Base de Schiff (salen) essa mesma atribuição é observada na faixa de $130-210{ }^{\circ} \mathrm{C}$ [EBRAHIMI et al., 2014]. Em $243^{\circ} \mathrm{C}$ e $365^{\circ} \mathrm{C}$ a decomposição da amina e dos aromáticos e em $507^{\circ} \mathrm{C}$ a saída do ligante. Na curva termogravimétrica do material mesoporoso SBA-15 foi observado um evento de perda de massa em $92{ }^{\circ} \mathrm{C}$ associado à eliminação de água fisissorvida. Na SBA-15 funcionalizada com 3-cloropropiltrimetóxisilano foram observados dois eventos: a eliminação da água fisissorvida em $144{ }^{\circ} \mathrm{C}$ e uma nova perda de massa em $300{ }^{\circ} \mathrm{C}$ atribuída a molécula orgânica ancorada na superfície do material relatados por [SOUNDIRESSANE et al., 2007], comprovando o sucesso de organofilização. No material [Ni(L1)]SBA-15, foram observados quatro eventos de decomposição: o primeiro em $167{ }^{\circ} \mathrm{C}$ atribuída a eliminação da água fisissorvida e solvente no material mesoporoso [BAGHERZADEH et al., 2014]. A decomposição da amina em $282{ }^{\circ} \mathrm{C}$ e $428^{\circ} \mathrm{C}$ e a saída do ligante foi observada em $671^{\circ} \mathrm{C}$ [ANACONA et al., 2014]. No [Ni(L2)]SBA-15, o primeiro evento ocorreu abaixo de $70^{\circ} \mathrm{C}$ sendo atribuída a eliminação de água fisissorvida e solvente no material mesoporoso. Em $300^{\circ} \mathrm{C}$ e $480^{\circ} \mathrm{C}$ foi observada a decomposição de amina e aromático e a decomposição térmica do ligante em $666{ }^{\circ} \mathrm{C}$, confirmando a ancoragem do complexo na sílica [ANACONA et al., 2014].

Ao se fazer uma comparação na temperatura, em relação à saída dos ligantes, nos complexos livres $[\mathrm{Ni}(\mathrm{L} 1)]-489^{\circ} \mathrm{C}, \quad[\mathrm{Ni}(\mathrm{L} 2)]-507{ }^{\circ} \mathrm{C}$ e nos complexos ancorados com o material mesoporoso [Ni(L1)]SBA- $15-671^{\circ} \mathrm{C}$ e $[\mathrm{Ni}(\mathrm{L} 2)] \mathrm{SBA}-15-666^{\circ} \mathrm{C}$, foi observada a degradação dos ligantes em temperaturas menores do que quando ancorados com a SBA-15, comprovando o aumento da estabilidade térmica do material híbrido inorgânico- orgânico.
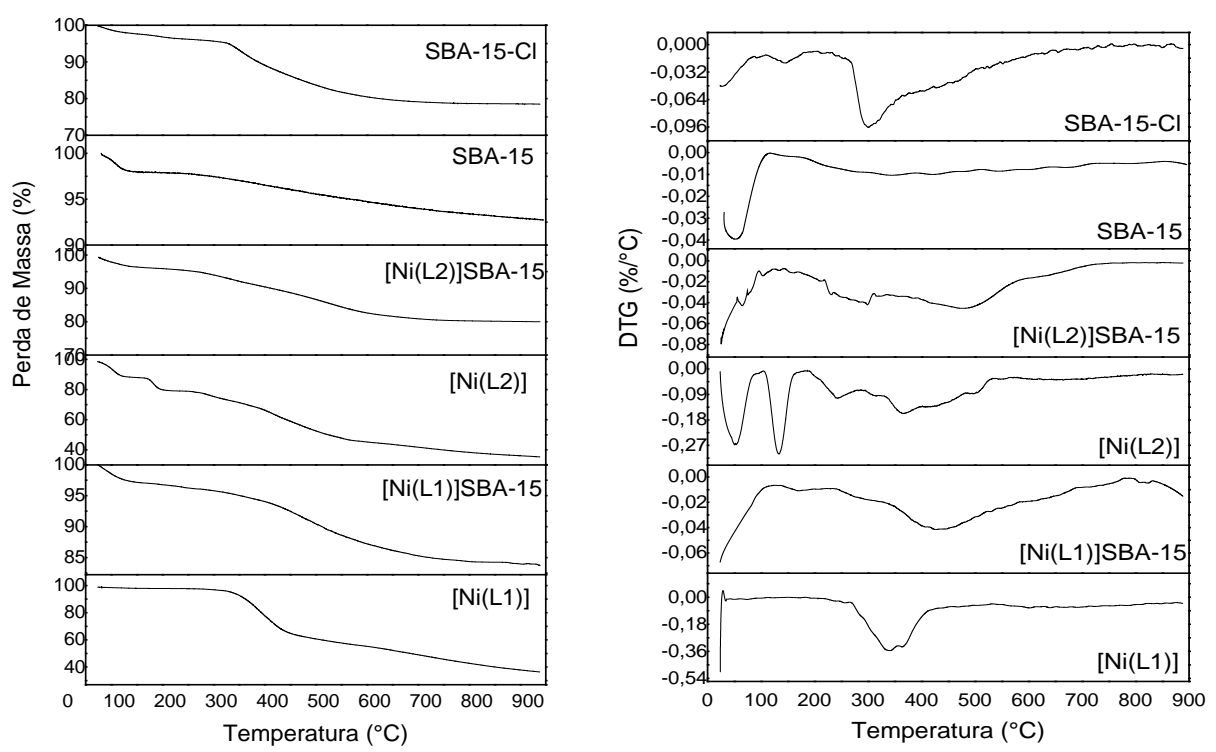

Figura 2 - TG e DTG dos materiais sintetizados 
Tabela 3 - Dados da analise térmica dos complexos, SBA-15, SBA-15-Cl e SBA-15 ancoradas com complexos.

\begin{tabular}{|c|c|c|c|c|c|}
\hline Compostos & $\begin{array}{c}\text { Estágios de } \\
\text { decomposição }\end{array}$ & $\begin{array}{c}\text { Temperatura } \\
\left({ }^{\circ} \mathrm{C}\right)\end{array}$ & T máx & $\begin{array}{c}\text { Perda de } \\
\text { Massa } \\
(\%)\end{array}$ & Atribuições \\
\hline$[\mathrm{Ni}(\mathrm{L} 1)]$ & $\begin{array}{l}1 \\
2\end{array}$ & $\begin{array}{r}47-183 \\
273-349 \\
\\
354-500 \\
465-569\end{array}$ & $\begin{array}{c}51 \\
338 \\
\\
\\
362 \\
489\end{array}$ & $\begin{array}{l}2,3 \\
8,4\end{array}$ & $\begin{array}{l}\text { Água fis. } \\
\text { Amina, } \\
\text { Aromático } \\
\text { acetato } \\
\text { amina } \\
\text { Ligante }\end{array}$ \\
\hline [Ni(L2)] & $\begin{array}{l}1 \\
2 \\
3 \\
4 \\
4\end{array}$ & $\begin{array}{c}23-98 \\
98-180 \\
190-295 \\
\\
295-603 \\
495-695\end{array}$ & $\begin{array}{c}52 \\
134 \\
243 \\
\\
375 \\
507\end{array}$ & $\begin{array}{l}10,5 \\
8,9 \\
7,2 \\
\\
28,3 \\
6,8\end{array}$ & $\begin{array}{c}\text { Água hidratação. } \\
\text { Água coord. } \\
\text { Amina } \\
\text { aromático } \\
\text { Amina } \\
\text { ligante }\end{array}$ \\
\hline SBA-15 & 1 & $71-160$ & 92 & 2,1 & Água fis. \\
\hline SBA-15-Cl & $\begin{array}{l}1 \\
2\end{array}$ & $\begin{array}{l}110-192 \\
216-700\end{array}$ & $\begin{array}{l}144 \\
300\end{array}$ & $\begin{array}{c}1,3 \\
17,3\end{array}$ & $\begin{array}{l}\text { Água fis. } \\
\text { 3-CPTMS }\end{array}$ \\
\hline $\begin{array}{c}{[\mathrm{Ni}(\mathrm{L} 1) \mathrm{SBA}-} \\
15\end{array}$ & $\begin{array}{l}1 \\
2 \\
2 \\
3 \\
4\end{array}$ & $\begin{array}{l}121-236 \\
236-330 \\
330-765 \\
593-816\end{array}$ & $\begin{array}{l}167 \\
\\
282 \\
428 \\
671\end{array}$ & $\begin{array}{c}2,06 \\
\\
1,49 \\
10,15 \\
8,06\end{array}$ & $\begin{array}{l}\text { Água fis. } \\
\text { Amina } \\
\text { Amina } \\
\text { ligante }\end{array}$ \\
\hline $\begin{array}{l}{[\mathrm{Ni}(\mathrm{L} 2)]} \\
\mathrm{SBA}-15\end{array}$ & $\begin{array}{l}1 \\
2 \\
3 \\
4 \\
5\end{array}$ & $\begin{array}{r}54-93 \\
\begin{array}{r} \\
93\end{array}-147 \\
164-314 \\
390-575 \\
596-822\end{array}$ & $\begin{array}{l}105 \\
300 \\
480 \\
666\end{array}$ & $\begin{array}{c}1,25 \\
\\
0,52 \\
4,12 \\
8,88 \\
7,6\end{array}$ & $\begin{array}{c}\text { Água fis. } \\
\text { Água coord. } \\
\text { Amina/aromático } \\
\text { Amina } \\
\text { ligante }\end{array}$ \\
\hline
\end{tabular}

Água fis: água fisissorvida / Água coord = água coordenada

\section{Conclusão}

De acordo com a análise elementar ( $\mathrm{CHN})$ a fórmula molecular sugerida para os complexos: [ $\left.\mathrm{Ni}\left(\mathrm{C}_{18} \mathrm{H}_{19} \mathrm{~N}_{3} \mathrm{O}_{2}\right)\right] \cdot 2 \mathrm{CH}_{3} \mathrm{COO} . \mathrm{H}_{2} \mathrm{O}$, [ $\left.\mathrm{Ni}\left(\mathrm{C}_{20} \mathrm{H}_{23} \mathrm{~N}_{3} \mathrm{O}_{2}\right)(2 \mathrm{Cl})\right] .2 \mathrm{H}_{2} \mathrm{O}$, sendo L1= $\mathrm{C}_{18} \mathrm{H}_{19} \mathrm{~N}_{3} \mathrm{O}_{2}, \mathrm{~L} 2=\mathrm{C}_{20} \mathrm{H}_{23} \mathrm{~N}_{3} \mathrm{O}_{2}$. Nos difratogramas de Raios-X, observamos que mesmo após o ancoramento o material não perdeu sua estrutura hexagonal ordenada. $\mathrm{O}$ decréscimo na área superficial, volume de poros, diâmetro de poros quando comparadas com a da SBA-15 confirmam a presença de complexos nos poros da sílica. Na Análise Termogravimétrica (TG/DTG) constatamos uma maior estabilidade térmica do complexo [Ni(L2)] quando ancorado na SBA-15. 


\section{Referências}

ABO-ALY, M.M., SALEM, A.M., SAYED, M.A., ABDEL AZIZ, A.A. Spectroscopy and structural studies of the Schiff base 3-methoxy-N-salicylidene-o-aminophenol complexes with some transition metal ions and their antibacterial, antifungal activities. Spectrochimica Acta Part A: Molecular and Biomolecular Spectroscopy, v.136, p.993 - 1000, 2015.

ALAVI, S., MONFARED, H.H., SICZEK, M. A new manganese (II) complex anchored onto SBA-15 as efficient catalyst for selective oxidation of cycloalkanes and cyclohexene with hydrogen peroxide. Journal of Molecular Catalysis A: Chemical, v. 377, p. 16 -28, 2013.

ANACONA, J.R., RODRIGUEZ, L.J., CAMUS, J. Synthesis, characterization and antibacterial activity of a Schiff Base derived from cephalexin and sulphatiazole and its transition metal complexes. Spectrochimica Acta Part A: Molecular and Biomolecular Spectroscopy, v.129, p. 96 - 102, 2014.

BAGHERZADEH, M., ZARE M., SALEMNOUSH T., OZKAR S., AKBAYRA S. Immobilization of dioxomolybdenum(VI) complex bearing salicylidene 2-picoloyl hydrazone on chloropropyl functionalizedSBA-15: A highly active, selective and reusable catalyst in olefin epoxidation. Applied Catalysis A: General, v. 475, p. 55-62, 2014.

DAVID, H.L., IONASHIRO, M., BENEDETTÌ, A.V., ZAMIAN, J.R., DOCKAL, E.R. Thermal decomposition of vanadyl Schiff -base compounds. Termochimica Acta, v.202, p. 45-50. 1992.

EBRAHIMI, P.H., HADI, S.J., ABDULNABI, A.Z., BOLANDNAZAR,Z. Spectroscopic, thermal analysis and DFT computational studies of salen-type Schiff base complexes. Spectrochimica Acta Part A: Molecular and Biomolecular Spectroscopy, v 117, p.485 - 492, 2014.

GALLO, T.O. Desenvolvimento de Peneiras Moleculares Mesoporosas do tipo MCM-41 e MCM-48 impregnadas com aminas para utilização na adsorção de $\mathrm{CO}_{2}$. Dissertação. Departamento de Química, Sergipe, 2012.

GHUA, A., BANU, S.K., DAS,S., CHATTOPADDHYAY,T., SANYAL, R., ZANGRANDO, E., DAS, D. A series of mononuclear nickel(II) complexes of Schiff-base ligands having N,N,Oand N,N,N-donor sites: Syntheses, crystal structures, solid state thermal property and catecholaselike activity. Polyhedron,v. 52, p. 669 - 678, 2013.

GRIVANI, G., BRUNO G., RUDBARI, H.A., KHALAJI, A.D., POURTEIMOURI, P. Synthesis, characterization and Crystal structure determination of a new oxovanadium (IV) Schiff base complex: The catalytic activity in the epoxidation of cyclooctene. Inorganic Chemistry Comunications, v.18, p. 15 - 20, 2012.

SHERINGTON, D.C in: B.K. HODNETT, A.P. KEYBETT, J.H. CLARK,. SMITH, K (Eds.), Supported Reagents and Catalyst in Chemistry, Royal Society of Chemistry, Cambridge, p. 220-228. 1998.

SILVA,C.M., SILVA, D.L., MODOLO, L.V., ALVES, R.B., RESENDE, M.A., MARTINS, C.V.B., ANGELO DE FATIMA. Schiff Base: A short review of their antimicrobial activities. Journal of Advanced Research, v.2, p.1 - 8, 2011 
SOUNDIRESSANE, T., SELVAKUMAR, S., MÉNAGE, S., HAMELIN, O., FONTECAVE, M., SINGH, A. P. Ru- and Fe-based N,N'-bis(2-pyridylmethyl)-N-methyl-(1S,2S)-1,2cyclohexanediamine complexes immobilized on mesoporous MCM-41: Synthesis, characterization and catalytic applications. Journal of Molecular Catalysis A: Chemical, v. 270, p. $132-143,2007$.

VEISI H., KORDESTAINI D., HEMMATI S., FARAJI A R., VEISI H. Catalytic applications of an organosuperbase dendron grafted on mesoporous SBA-15 and a related palladium complex in Henry and Suzuki-Miyaura coupling reactions. Tetrahedron Letters, v. 55, p. 5311-5314. 2014

YANG, Y., ZHANG, Y., HAO, S., KAN, Q. Terthering of Cu(II), Co(II) and Fe(II) tetrahydro salen and salen complexes onto amino - functionalizated SBA-15. Effects of salen ligand hydrogenation on catalytic performances for aerobic epoxidation of styrene. Chemical Engineering Journal, China, v. 171, p. 1356 - 1366, 2011

WANG, J., GE H., BAO W. Synthesis and characteristics of SBA-15 with thick pore wall and high hydrothermal stability. Materials Letters, v.145, p. 312-315. 2015

ZHAO, D., FENG, J.,HUO, Q., MOLISH, N., FREDRICKSON, G.H., B.F. CHMELKA, B.F., STUCKY, G.D. Triblock copolymer syntheses of mesoporous sílica with periodic 50 to $300 \AA$ pores. Science, v. 279, p. 548, 1998 a.

ZHAO, D.,.HUO, Q., FENG, J., CHMELKA, B.F., STUCKY, G.D. Nonionic triblock and Star Diblock copolymer and Oligomeric Surfactant Syntheses of Highly Ordered Hydrothermally Stable, Mesoporous Sílica Structures. Journal of American Chemical Society, v.120, p.60246036, 1998 b. 